Check for updates

Cite this: RSC Adv., 2019, 9, 33872

Received 24th August 2019 Accepted 16th October 2019

DOI: 10.1039/c9ra06671c

rsc.li/rsc-advances

\section{Silkworm cocoon derived N, O-codoped hierarchical porous carbon with ultrahigh specific surface area for efficient capture of methylene blue with exceptionally high uptake: kinetics, isotherm, and thermodynamics $\dagger$}

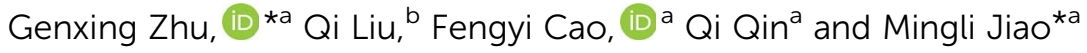

Dyes are typical water contaminants that seriously affect water quality. In this study, silkworm cocoon derived N, O-codoped hierarchical porous carbon was successively developed via a facile precarbonization and chemical activation method, and characterized thoroughly by SEM, TEM, HRTEM, XRD, Raman, $\mathrm{N}_{2}$ adsorption and XPS. The as-prepared N, O-HPC showed a well-developed porous structure with an ultra-high specific surface area of $2270.19 \mathrm{~m}^{2} \mathrm{~g}^{-1}$, which proved to be a highefficiency adsorbent. Batch adsorption experiments demonstrated that MB adsorption was highly dependent on contact time, initial MB concentration, temperature and initial solution $\mathrm{pH}$. However, no remarkable effects of humic acid and ionic strength were observed. In the kinetic studies, the good applicability of a pseudo-second-order kinetic model was demonstrated. The adsorption isotherm study showed that a Langmuir isotherm model can describe the experimental data much more suitably with a maximum monolayer adsorption capacity value of $2104.29 \mathrm{mg} \mathrm{g}^{-1}$, which is among the highest in previously reported adsorbents and ascribed to multiple adsorption mechanisms including pore filling, $\pi-\pi$ stacking interaction and electrostatic interaction between MB and N, O-HPC. Thermodynamic analyses suggested that MB adsorption onto N, O-HPC was spontaneous and endothermic. Furthermore, the as prepared adsorbent showed highly efficient adsorption for $M B$ in tap water and synergistic adsorption performance toward MB and MO. Therefore, N, O-HPC derived from silkworm cocoon could be considered as an efficient, novel and advantageous material for wastewater remediation.
\end{abstract}

\section{Introduction}

Nowadays, dyes are widely used in the textile, leather, papermaking, dye synthesis, printing, food, cosmetics and plastics industries. ${ }^{1}$ Water contamination caused by these industries has drawn more and more attention, since almost all dyes are poorly biodegradable or resistant to environmental conditions and, therefore, create major problems in the treatment of wastewater stemming from the dyeing industry. ${ }^{2}$ Currently, various treatment methods including physical, chemical and biological schemes have been developed to remove dyes from wastewater, such as photocatalytic degradation, ${ }^{3-6}$ electrochemical degradation, ${ }^{7,8}$ photochemical degradation, ${ }^{9,10}$

${ }^{a}$ School of Materials and Chemical Engineering, Zhongyuan University of Technology, Zhengzhou, Henan 450007, P. R. China. E-mail: zgxzjucsufydxx@zzti.edu.cn; johnml@163.com

${ }^{b}$ College of Science, Zhongyuan University of Technology, Zhengzhou, Henan 450007, P. R. China

$\dagger$ Electronic supplementary information (ESI) available. See DOI: $10.1039 / \mathrm{c} 9 \mathrm{ra} 06671 \mathrm{c}$ sonochemical degradation, ${ }^{11-13}$ sonocatalytic degradation, ${ }^{14,15}$ Fenton-like degradation, ${ }^{16-18}$ photo-Fenton degradation, ${ }^{19-21}$ advanced oxidation process, ${ }^{22-25}$ electro-Fenton degradation ${ }^{26,27}$ and adsorption. ${ }^{28-35}$ Among those, adsorption is proven to be a potentially powerful technique for removing pollutants from contaminated aqueous media. It is preferred over other methods because of its relative simplicity of design, ease of operation, cost effectiveness, insensitivity to noxious pollutants, energy efficiency and environmental benignity. ${ }^{36-39}$ Numerous adsorbents, such as MOF-based material, ${ }^{33}$ geopolymers, ${ }^{31}$ graphene-based material, ${ }^{30,32}$ palygorskite and its composites, ${ }^{40,41}$ clay-based minerals, ${ }^{42,43}$ agricultural peels, ${ }^{44}$ hydrogels ${ }^{45-47}$ carbon nanotubes ${ }^{48}$ etc have been studied. Among the adsorbents mentioned, the carbonaceous materials usually have the highest dye removal efficiency due to their large surface area and the functional groups on their surface that facilitate interactions with various dyes. ${ }^{1}$

Methylene blue (MB) is a cationic dye that is most commonly used for colouring. It is generally used for dyeing cotton, wool, and silk. MB can cause eye burns in humans and animals, methemoglobinemia, cyanosis, convulsions, tachycardia, 
dyspnea, irritation to the skin, and if ingested, irritation to the gastrointestinal tract, nausea, vomiting, and diarrhea. ${ }^{49}$ Therefore, MB often serves as a model compound for removing organic contaminants and coloured bodies from aqueous solutions.

Biomass materials are promising precursors that can be used to prepare hierarchically porous carbons with high surface area and rich heteroatom doping. As a natural bio-polymer, silkworm cocoon is mainly composed of protein with fibroin being enveloped by the glue-like sericin, and the rich heteroatoms including $\mathrm{N}$ atoms is beneficial for the preparation of nitrogen-doped carbons during pyrolysis. ${ }^{50}$ Moreover, the trace inorganic salts dispersing in the silk is a natural template that is favourable for the homogeneously creation and expansion of pores through adjusting the activation condition during pyrolysis, facilitating the formation of hierarchically porous structure. ${ }^{51}$

Herein, we reported a successful synthesis of N, O-codoped hierarchical porous carbon (N, O-HPC) with ultrahigh specific surface area by a pre-carbonization and a subsequent $\mathrm{KOH}$ activation of silkworm cocoon. The as-prepared N, O-HPC with an ultrahigh specific surface area of $2270.19 \mathrm{~m}^{2} \mathrm{~g}^{-1}$ proved to be novel and highly efficient adsorbent for MB. The effects of contact time, initial $\mathrm{MB}$ concentration, temperature, initial solution $\mathrm{pH}$, adsorbent dosage, ionic strength and co-existing humic acid were systematically investigated. The adsorption process fitted well with the Langmuir isotherm model and pseudo-second-order kinetic model, and the maximum adsorption capacity was $2104.29 \mathrm{mg} \mathrm{g}^{-1}$. To the best of our knowledge, this is among the highest in the previously reported adsorbents. Moreover, MB adsorption onto N, O-HPC was spontaneous and endothermic, and the as prepared adsorbent showed highly efficient adsorption for $\mathrm{MB}$ in tap water and synergistic adsorption performance toward $\mathrm{MB}$ and $\mathrm{MO}$. Therefore, N, O-HPC derived from silkworm cocoon is expected to be a promising adsorbent for the efficient removal of dyes from wastewater.

\section{Experimental}

\subsection{Materials}

The raw silkworm cocoon was directly obtained from sericulturists of Sichuan, China. Potassium hydroxide $(\mathrm{KOH})$ were purchased from Aladdin Chemistry Co. Ethanol absolute and hydrochloric acid (37 wt\%, $\mathrm{HCl}$ ) were purchased from Sinopharm Chemical Reagent Co., Ltd. Methylene blue (MB) was purchased from Shanghai Macklin Biochemical Co., Ltd. All the chemicals used in the sample preparation were used as received without further purification. Furthermore, deionized water was used to prepare different concentrations of MB solutions.

\subsection{Preparation of $\mathrm{N}$, O-codoped hierarchical porous carbon} (N, O-HPC)

In a typical process, after cut into pieces, silkworm cocoon was washed and dried at $80{ }^{\circ} \mathrm{C}$ for $48 \mathrm{~h}$. Subsequently, the above dry and clean precursor was loaded into a corundum crucible and pre-carbonized in a horizontal tubular furnace at $450{ }^{\circ} \mathrm{C}$ for $0.5 \mathrm{~h}$ with a heating rate of $5^{\circ} \mathrm{C} \min ^{-1}$ under $\mathrm{N}_{2}$ flow. After cooling down to room temperature, the resulting carbon material was ground into fine powder, which was thoroughly impregnated in $\mathrm{KOH}$ aqueous solution at a mass ratio of $\mathrm{C}: \mathrm{KOH}=1: 1$ and dehydrated at $80{ }^{\circ} \mathrm{C}$ to form a slurry. Afterward, the mixture was transferred into a corundum crucible and activated in a tubular furnace at $900{ }^{\circ} \mathrm{C}$ for $2 \mathrm{~h}$ with a heating rate of $2{ }^{\circ} \mathrm{C}$ min $^{-1}$ under $\mathrm{N}_{2}$ flow. After being cooled to ambient temperature, the activated product was adjusted to $\mathrm{pH}$ $\approx 7$ by $1 \mathrm{M} \mathrm{HCl}$ solution and magnetically stirred for several hours. After that, the sample was centrifuged and repeatedly washed abundantly with deionized water and finally dried at $80{ }^{\circ} \mathrm{C}$ overnight to obtain the final product (denoted as $\mathrm{N}$, OHPC).

\subsection{Characterization of N, O-HPC}

Scanning electron microscopy (SEM) measurements were conducted on a Hitachi S-4800 field emission scanning electron microscope. Transmission electron microscopy (TEM) and high-resolution transmission electron microscopy (HRTEM) images were taken from a JEM-2100F (field emission) scanning transmission electron microscope (JEOL, Japan) with an acceleration voltage of $200 \mathrm{kV}$. X-ray powder diffraction (XRD) pattern was recorded on a Rigaku Dmax 2200 diffractometer with $\mathrm{Cu} \mathrm{K} \alpha 1$ radiation $(\lambda=1.5416 \AA$, operating at $40 \mathrm{kV}$ and 40 $\mathrm{mA}$ ). Raman spectrum was recorded on a LABRAM-HR confocal laser micro-Raman spectrometer (Jobin-Yvon, France) in the backscattering configuration excited with an $\mathrm{Ar}+$ ion laser $(\lambda=$ $514.5 \mathrm{~nm}$ ). X-ray photoelectron spectroscopy (XPS) analyses were measured using an ESCALAB 250Xi X-ray photoelectron spectrometer with $\mathrm{Al} \mathrm{K} \alpha$ monochromatic radiation at a constant pass energy of $1486.6 \mathrm{eV}$ (ThermoFisher Scientific, USA). The nitrogen adsorption-desorption isotherms were carried out by $\mathrm{N}_{2}$ physisorption at $77 \mathrm{~K}$ on an automatic Micromeritics ASAP 2020M nitrogen adsorption analyzer, and with the adoption of the Brunauer-Emmett-Teller (BET) model and nonlocal density functional theory (NLDFT) methods to calculate the specific surface area (SSA) and the pore size distribution (PSD). Fourier transform infrared (FTIR) spectra were obtained using a FTIR spectrometer (IRAffinity-1, Shimadzu, Japan).

\subsection{Adsorption experiments}

Batch adsorption experiments was conducted in a set of $200 \mathrm{~mL}$ glass beakers containing $100 \mathrm{~mL} \mathrm{MB}$ solutions at a consistent stirring rate. The initial solution $\mathrm{pH}$ was adjusted by using $0.1 \mathrm{M}$ $\mathrm{NaOH}$ or $0.1 \mathrm{M} \mathrm{HCl}$ solution. Typically, the adsorption kinetics was investigated at $293 \mathrm{~K}$ by adding $0.05 \mathrm{~g} \mathrm{~N}$, O-HPC into $700 \mathrm{mg} \mathrm{L}^{-1} \mathrm{MB}$ solution without adjusting $\mathrm{pH}$ value. The adsorption isotherm experiments were carried out at $293 \mathrm{~K}$ by adding $0.05 \mathrm{~g} \mathrm{~N}$, O-HPC into different MB solutions with various initial concentrations (400-1000 $\mathrm{mg} \mathrm{L}^{-1}$ ) and without adjusting $\mathrm{pH}$. The adsorption thermodynamics was studied by conducting the adsorption experiment at $293 \mathrm{~K}, 303 \mathrm{~K}$ and $313 \mathrm{~K}$. The effects of experimental parameters such as N, O-HPC dosage, initial solution $\mathrm{pH}$, ionic strength and humic acid on the 
adsorption process were also investigated. Moreover, the adsorption of $\mathrm{MB}$ in tap water and synergistic adsorption performance toward MB and MO were also evaluated. At predetermined time intervals, $1 \mathrm{~mL} \mathrm{MB}$ solution was withdrawn and filtrated using a $0.22 \mu \mathrm{m}$ syringe filter, and then measured by UV-vis spectrophotometer at its maximum wavelength of $664 \mathrm{~nm}$. The amount of MB adsorbed $q_{t}$ and the dye removal efficiency $(R)$ at any time $t$, was calculated using the following equations:

$$
\begin{gathered}
q_{t}=\frac{\left(C_{0}-C_{t}\right) V}{m} \\
R=\frac{\left(C_{0}-C_{t}\right) \times 100}{C_{0}}
\end{gathered}
$$

where $C_{0}$ and $C_{t}\left(\mathrm{mg} \mathrm{L}^{-1}\right)$ are the liquid-phase concentrations of $\mathrm{MB}$ at initial and any time $t$, respectively. $V(\mathrm{~L})$ is the volume of MB solution, and $m(\mathrm{~g})$ is the mass of dry adsorbent used.

\subsection{Determination of point of zero charge (pHpzc)}

For the determination of pHpzc of the adsorbent, $10 \mathrm{~mL}$ of $0.01 \mathrm{M} \mathrm{NaCl}$ solution was added into a series of $20 \mathrm{~mL}$ capped glass bottles. The initial $\mathrm{pH}\left(\mathrm{pH}_{\mathrm{i}}\right)$ of solutions was adjusted between 2 to 10 by using $0.1 \mathrm{M} \mathrm{HCl}$ or $0.1 \mathrm{M} \mathrm{NaOH}$. Then, $10 \mathrm{mg}$ of N, O-HPC was added to each glass bottle, and the obtained suspensions were shaken in controlled temperature $\left(20 \pm 2{ }^{\circ} \mathrm{C}\right)$ with a speed of $150 \mathrm{rpm}$. After $48 \mathrm{~h}$, the suspensions were filtered and final $\mathrm{pH}$ values $\left(\mathrm{pH}_{\mathrm{f}}\right)$ of the filtrates were measured again. The difference between $\mathrm{pH}_{\mathrm{i}}$ and $\mathrm{pH}_{\mathrm{f}}$ values $\left(\Delta \mathrm{pH}=\mathrm{pH}_{\mathrm{i}}-\right.$ $\mathrm{pH}_{\mathrm{f}}$ ) was plotted versus the $\mathrm{pH}_{\mathrm{i}}$. The point of intersection of the resulting curve with abscissa, at which $\Delta \mathrm{pH}=0$, provided the point of zero charge. ${ }^{52,53}$

\subsection{Adsorption kinetics}

The Lagergren pseudo-first order, ${ }^{54}$ pseudo-second order $^{55}$ and intra-particle diffusion ${ }^{56}$ models were used to describe the experimental adsorption kinetic data. The Lagergren pseudofirst-order equation is expressed as:

$$
q_{t}=q_{\mathrm{e}}\left[1-\exp \left(-k_{1} t\right)\right]
$$

where $q_{\mathrm{e}}$ and $q_{t}$ are the amount of adsorbate adsorbed ( $\mathrm{mg} \mathrm{g}^{-1}$ ) at equilibrium and at time $t$, respectively. $t$ is the contact time (min) and $k_{1}$, the rate constant $\left(\min ^{-1}\right)$.

The pseudo-second order model, proposed by Ho and McKay, can be represented in the following linear form:

$$
\frac{t}{q_{t}}=\frac{t}{q_{\mathrm{e}}}+\frac{1}{k_{2} q_{\mathrm{e}}^{2}}
$$

where $k_{2}$ is the pseudo-second-order rate constant ( $\mathrm{g}$ $\left.\mathrm{mg}^{-1} \min ^{-1}\right) ; k_{2} q_{\mathrm{e}}{ }^{2}$ is the initial adsorption rate $\left(\mathrm{mg} \mathrm{g}^{-1}\right)$. The values of $q_{\mathrm{e}}$ and $k_{2}$ were obtained by a linear plot $t / q_{t}$ versus $t$.

The intraparticle diffusion model equation can be written as:

$$
q_{t}=k_{\text {intra }} \sqrt{t}+C
$$

where $k_{\text {intra }}$ is the intraparticle diffusion rate constant $\left(\mathrm{mg} \mathrm{g}^{-1}\right.$ $\left.\min ^{-0.5}\right)$ and $\mathrm{C}$ represents the intercept $\left(\mathrm{mg} \mathrm{g}^{-1}\right)$. A plot of $q_{t}$ versus $t^{0.5}$ should be in straight line if the adsorption process follows the intraparticle diffusion; meanwhile if the line passes through the origin then intra particle diffusion will be the sole controlling step.

\subsection{Adsorption isotherms}

In this study, the equilibrium adsorption data were analysed by Langmuir, ${ }^{57,58}$ Freundlich $^{59}$ and Temkin ${ }^{60}$ models. Langmuir isotherm model has been successfully applied to many adsorption processes. It assumes that adsorption occurs at specific homogenous sites within the adsorbent. Once a molecule occupies a site, no further adsorption will take place at the site. The linear form of the Langmuir isotherm equation can be represented by the following equation:

$$
\frac{C_{\mathrm{e}}}{q_{\mathrm{e}}}=\frac{C_{\mathrm{e}}}{q_{\mathrm{m}}}+\frac{1}{k_{\mathrm{L}} q_{\mathrm{m}}}
$$

where $C_{\mathrm{e}}\left(\mathrm{mg} \mathrm{mL}^{-1}\right)$ is the equilibrium concentration of adsorbate in the solution; $q_{\mathrm{m}}\left(\mathrm{mg} \mathrm{g}^{-1}\right)$, the maximum adsorption capacity that corresponds to complete monolayer coverage, and $k_{\mathrm{L}}$, the equilibrium constant $\left(\mathrm{mL} \mathrm{g}^{-1}\right)$.

The essential characteristics of the Langmuir isotherm can be expressed by the dimensionless constant separation factor $\left(R_{\mathrm{L}}\right)$ which is given by:

$$
R_{\mathrm{L}}=\frac{1}{1+k_{\mathrm{L}} C_{0}}
$$

The adsorption process can be defined as irreversible $\left(R_{\mathrm{L}}=\right.$ 0 ), favorable $\left(R_{\mathrm{L}}\right.$ between 0 and 1$)$, linear $\left(R_{\mathrm{L}}=1\right)$ or unfavorable $\left(R_{\mathrm{L}}>1\right)$.

The Freundlich isotherm assumes a heterogeneous system with different energies of active sites and reversible adsorption, which is not restricted to monolayer sorption. It is an empirical equation which is used for the description of multilayer adsorption with interaction between adsorbed molecules. The Freundlich isotherm is shown as below:

$$
\log q_{\mathrm{e}}=\log k_{\mathrm{F}}+\frac{1}{n} \log C_{\mathrm{e}}
$$

where $k_{\mathrm{F}}\left(\mathrm{mg} \mathrm{g}^{-1}\right)$ is Freundlich constant representing the adsorption capacity; $n$ (dimensionless), an empirical parameter related to the adsorption intensity. The value of $n$ varies with the heterogeneity of the adsorbent and for favourable adsorption process the value of $n$ should lie in the range of 1-10.

Temkin isotherm assumes that the heat of adsorption of all the molecules in the layer would decrease linearly with coverage and the adsorption is characterized by a uniform distribution of binding energies, up to some maximum binding energy. The Temkin isotherm equation is as follow:

$$
q_{\mathrm{e}}=\frac{R T}{b} \ln A_{\mathrm{T}}+\frac{R T}{b} \ln C_{\mathrm{e}}
$$

where $R$ is the gas constant; $T$, the absolute temperature; $A_{\mathrm{T}}(\mathrm{L}$ $\mathrm{mg}^{-1}$ ), the Temkin equilibrium binding constant and $b$ (dimensionless), the Temkin isotherm constant. 


\subsection{Adsorption thermodynamics}

Thermodynamics parameters including enthalpy change $\left(\Delta H^{0}\right)$, Gibbs free energy change $\left(\Delta G^{0}\right)$ and entropy change $\left(\Delta S^{0}\right)$ can be determined as follows: ${ }^{\mathbf{1 1}}$

$$
\begin{gathered}
\Delta G^{0}=\Delta H^{0}-T \Delta S^{0} \\
\Delta G^{0}=-R T \ln K_{\mathrm{d}} \\
K_{\mathrm{d}}=\frac{q_{\mathrm{e}}}{C_{\mathrm{e}}} \\
\ln K_{\mathrm{d}}=\frac{\Delta S^{0}}{R}-\frac{\Delta H^{0}}{R T}
\end{gathered}
$$

where $K_{\mathrm{d}}$ is the equilibrium constant; $q_{\mathrm{e}}\left(\mathrm{mg} \mathrm{g}^{-1}\right)$ is the amount of adsorbate that is adsorbed at equilibrium; $C_{\mathrm{e}}$ is equilibrium concentration $\left(\mathrm{mg} \mathrm{\textrm {L } ^ { - 1 }}\right)$ of adsorbate in the solution; $T$ is the absolute temperature $(\mathrm{K})$ and $R$ is the universal gas constant.

\section{Results and discussion}

\subsection{Characterization of N, O-HPC}

$\mathrm{N}$, O-HPC was successively developed via a facile precarbonization and chemical activation method (Scheme S1 $\dagger$ ). The SEM and TEM images of N, O-HPC exhibit their morphologies and porous structures. As shown in Fig. 1A and B, N, OHPC displays a loose and well-developed porous structure with many open cavities. Specifically, under high-resolution, N, O-HPC exhibits a typical honeycomb-like porous structure with abundant interconnected mesopores and macropores (Fig. 1C). TEM and HRETM images also reveal a porous structure for N, OHPC. As shown in Fig. 1D-F, the synthesized sample has an interconnected 3D framework and porous structure with highdensity macropores, mesopores and micropores. The highdensity pores and open cavities are apparently favourable for the quick transportation of adsorbate molecules. Promisingly, some bent lattice fringes with a spacing of about $0.34 \mathrm{~nm}$ can be observed in the HRTEM image (Fig. 1F), corresponding to (002) plane of the graphitic stacking, which indicates the partial graphitization of the prepared carbon sample. ${ }^{62}$

As shown in Fig. 2A, XRD pattern of N, O-HPC shows two broad diffraction peaks at $15-26^{\circ}$ and $40-47^{\circ}$ ranges, consistent with the graphitic carbon (JCPDS card file no. 75-1621), suggesting a turbostratic structure of carbon with low crystallinity. The broad diffraction peak centered at $\sim 23^{\circ}$ can be assigned to the (002) plane spacing corresponding to the interlayer spacing of the graphitic carbon, and the peak centered at $\sim 43^{\circ}$ can be attributed to a superposition of the (100) and (101) reflections of the interlayer condensation with a limited degree of graphitization. ${ }^{\mathbf{6 3 , 6 4}}$ Moreover, it is noticed that the pattern presented a significantly enhanced intensity in its small-angle region $(2 \theta<$ $15^{\circ}$ ), indicating the presence of high-density micropores, ${ }^{65}$ thus confirming the results of TEM studies.
The Raman spectrum (Fig. 2B) exhibits two prominent first-order band peaks and two weak second-order band peaks. The peak centered at $1350 \mathrm{~cm}^{-1}$ ( $\mathrm{D}$ band) is commonly assigned to the disordered or defective graphitic structure with the breathing mode of $k$-point phonons of $A_{1 \mathrm{~g}}$ symmetry; ${ }^{66}$ while the peak located at $1590 \mathrm{~cm}^{-1}$ (G band) is associated with the ordered graphitic structure with an $\mathrm{E}_{2 \mathrm{~g}}$ mode of graphite. ${ }^{67}$ As known, the D band should be originated from the vibration of carbon atoms with dangling bonds in planar terminations of disordered graphite; while the $\mathrm{G}$ band should stem from the tangential vibration of $\mathrm{sp}^{2}$ bonded carbon atoms in a two-dimensional hexagonal lattice. The $\mathrm{D}$ band implies the appearance of structural disorder in the carbon specimen owing to the porous structure and heteroatom doping. It is generally recognized that the intensity ratio of $\mathrm{D}$ to $\mathrm{G}$ band $\left(I_{\mathrm{D}} / I_{\mathrm{G}}\right)$ can be used to evaluate the degree of structural disorder in the carbon materials. Herein, the $I_{\mathrm{D}} / I_{\mathrm{G}}$ ratio of the prepared carbon can be calculated to be about 1.01, indicating the existence of abundant disordered carbon structures. Moreover, the full width at half maximum of the $\mathrm{D}$ band is distinctly wider than that of $\mathrm{G}$ band, revealing a relatively high proportion of defects and disorder in the resultant carbon matrix, which is in good accordance with the XRD result. Additionally, a large graphitic domain size of $4.31 \mathrm{~nm}$ can be calculated by using the formula $L_{\mathrm{a}}=4.35 \times\left(I_{\mathrm{G}} / I_{\mathrm{D}}\right) .{ }^{68}$ The larger domain size is usually advantageous for the higher surface reactivity of the carbon materials, and will promote the adsorption process by providing more unsaturated carbon atoms on the surface. Furthermore, the widening and weakening of the $2 \mathrm{D}$ band at around $2690 \mathrm{~cm}^{-1}$ and the combination band of $\mathrm{G}+\mathrm{D}$ at about $2940 \mathrm{~cm}^{-1}$ also can be observed. The $2 \mathrm{D}$ band and $(\mathrm{G}+$ D) band are ascribed to the two-phonon process activated by double resonance and the combination of phonons with different momentum around the $\Gamma$ and $K$ points, respectively. ${ }^{67,69}$ These observations further demonstrate that the as-prepared carbon possesses a partial graphitization structure and a mass of defects (heteroatom co-doping and porosity).

To better understand the porous structure of N, O-HPC, $\mathrm{N}_{2}$ adsorption-desorption and pore size distribution were performed as shown in Fig. 2C and D. As can be seen, the isotherm shows a combined Type I and IV with $\mathrm{H} 4$ hysteresis loop according to the International Union of Pure and Applied Chemistry (IUPAC) classification, indicating the coexistence of micropores and mesopores/macropores. The dramatic increase in the isotherm at a relatively low pressure $\left(P / P_{0}<0.2\right)$ can be attributed to the presence of high-density micropores. At medium and high relative pressure regions $\left(P / P_{0}=0.4-1.0\right)$, a small hysteresis loop associated with a capillary condensation step indicates the presence of mesopores and macropores. The specific surface area (SSA) is calculated to be $2270.19 \mathrm{~m}^{2} \mathrm{~g}^{-1}$, demonstrating a good activation effect caused by KOH. As welldemonstrated in the literature, ${ }^{\mathbf{7 0}, 71}$ the activation mechanism for the resulting $\mathrm{N}$, O-HPC with $\mathrm{KOH}$ might be explained as follows: (1) the etching of carbon by $\mathrm{KOH}$ and the resulting $\mathrm{K}_{2} \mathrm{CO}_{3} / \mathrm{K}_{2} \mathrm{O}$ through redox reactions $\left(6 \mathrm{KOH}+2 \mathrm{C} \rightarrow 2 \mathrm{~K}+3 \mathrm{H}_{2}+\right.$ 

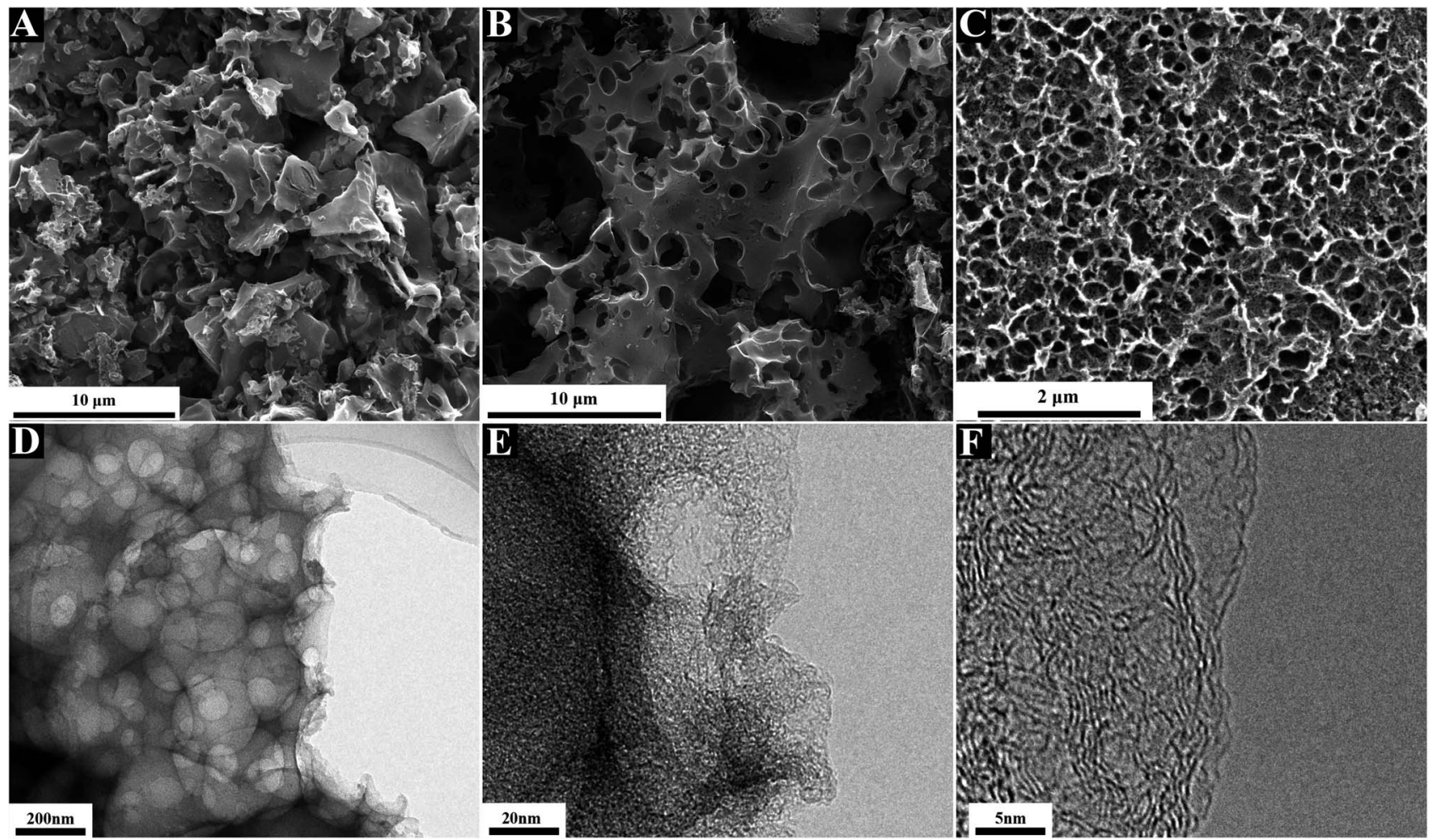

Fig. 1 (A-C) SEM, (D) TEM and (E and F) HRTEM images of N, O-HPC.

$2 \mathrm{~K}_{2} \mathrm{CO}_{3} ; \mathrm{K}_{2} \mathrm{CO}_{3} \rightarrow \mathrm{K}_{2} \mathrm{O}+\mathrm{CO}_{2} ; \mathrm{K}_{2} \mathrm{CO}_{3}+2 \mathrm{C} \rightarrow 2 \mathrm{~K}+3 \mathrm{CO} ; \mathrm{K}_{2} \mathrm{O}+\mathrm{C}$ $\rightarrow 2 \mathrm{~K}+\mathrm{CO}$ ); (2) the gasification of carbon by the formed water vapor and $\mathrm{CO}_{2}\left(\mathrm{H}_{2} \mathrm{O}+\mathrm{C} \rightarrow \mathrm{CO}+\mathrm{H}_{2} ; \mathrm{CO}_{2}+\mathrm{C} \rightarrow 2 \mathrm{CO}\right)$; and (3) the intercalation of the intermediate $\mathrm{K}$ into the carbon framework. Furthermore, the plot of pore size distribution shows that the sample primarily composes of mesopores with the pore size ranging from 3.5 to $5 \mathrm{~nm}$. The high SSA and well-developed hierarchically porous structure of N, O-HPC make it promising as ideal absorbent material for dye removal from coloured wastewater.
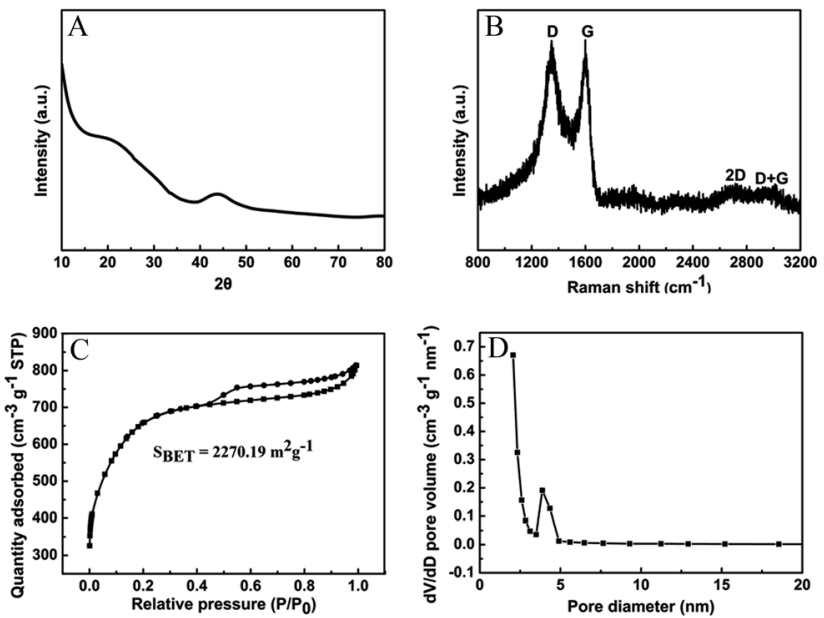

Fig. 2 (A) XRD pattern, (B) Raman spectrum, (C) $\mathrm{N}_{2}$ adsorptiondesorption isotherm and (D) pore size distribution of $\mathrm{N}, \mathrm{O}-\mathrm{HPC}$.
The chemical composition related to the surface functional groups of the prepared carbon materials were conducted by XPS measurement. As shown in Fig. 3A, the XPS survey scan of the sample showing obvious peaks of $\mathrm{C} 1 \mathrm{~s}, \mathrm{~N} 1 \mathrm{~s}$, and $\mathrm{O} 1 \mathrm{~s}$, indicating the successful synthesis of the in situ N, O-codoped hierarchical porous carbon. $86.93 \mathrm{wt} \% \mathrm{C}, 1.71 \mathrm{wt} \% \mathrm{~N}$ and $11.36 \mathrm{wt} \% \mathrm{O}$ were detected in the N, O-HPC sample. The high-
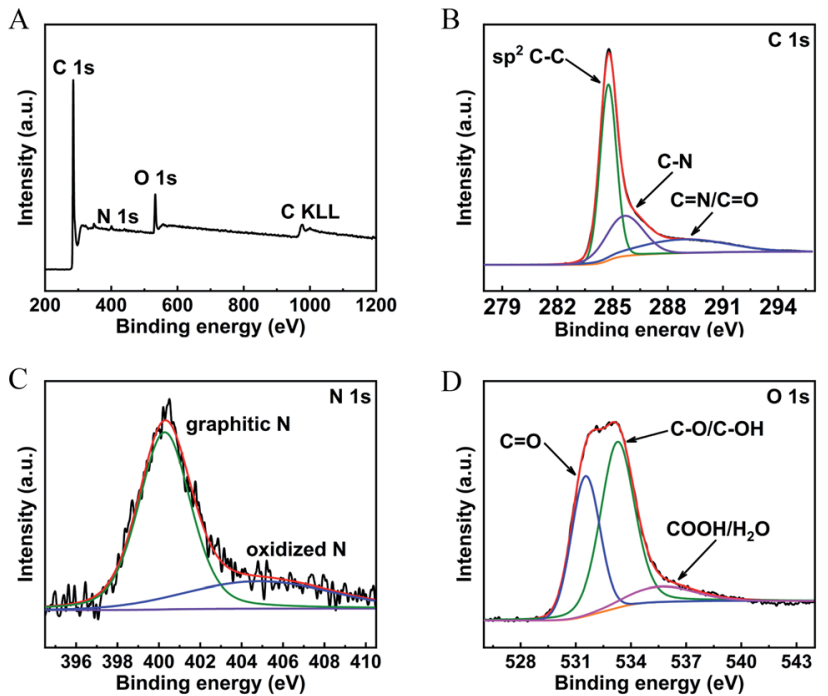

Fig. 3 (A) XPS survey spectrum and the high-resolution spectra for (B) $\mathrm{C} 1 \mathrm{~s},(\mathrm{C}) \mathrm{N}$ 1s and (D) O 1s together with the fitting peaks of $\mathrm{N}, \mathrm{O}-\mathrm{HPC}$. 
resolution $\mathrm{C}$ 1s spectrum (Fig. 3B) can be divided into three individual peaks, centered at about 284.8, 285.7, and $288.9 \mathrm{eV}$, corresponding to $\mathrm{C}-\mathrm{C}, \mathrm{C}-\mathrm{N}$ and $\mathrm{C}=\mathrm{O} / \mathrm{C}=\mathrm{N}$ binding. ${ }^{72,73}$ The high-resolution $\mathrm{N}$ 1s spectra shown in Fig. 3C can be deconvoluted into two peaks, centered at about 400.3 and $404.7 \mathrm{eV}$, which can be attributed to characteristic nitrogen functionalities of graphitic nitrogen and oxidized nitrogen. ${ }^{74,75}$ The highresolution $\mathrm{O}$ 1s spectrum (Fig. 3D) revealed the presence of three oxygen-based components, which can be resolved into three peaks at $531.5 \mathrm{eV}$ (keto oxygen or quinone group, O-I), $533.3 \mathrm{eV}$ (esters or phenolic group, O-II) and $535.5 \mathrm{eV}$ (carboxylic group or water, O-III), respectively. ${ }^{76}$

\subsection{Effect of contact time and adsorption kinetics}

The effect of contact time on the adsorption capacities of N, OHPC for MB was investigated. As shown in Fig. S1, $\dagger$ there was a rapid increase in $\mathrm{MB}$ absorbed amount in the first $15 \mathrm{~min}$, which could be ascribed to the presence of numerous adsorption active sites on the N, O-HPC surface. During the following period, the adsorption rate slowed down, which was arisen from: (1) a reduction in adsorption active sites present on the adsorbent surface; (2) occupancy of the pores in the adsorbent by $\mathrm{MB}$ molecules, leading to slower internal diffusion. ${ }^{77}$ After $45 \mathrm{~min}$, adsorption equilibrium was reached gradually, indicating that the adsorbents owned high adsorption efficiency towards MB.

The Lagergren pseudo-first order, pseudo-second order and intra-particle diffusion models were employed to describe the kinetics of MB adsorption. As shown in Fig. 4, it can be found that the regression coefficient $R^{2}$ obtained from the pseudo-second order model (0.9998) is much higher than those from other models, suggesting that the pseudo-second order model fits better with the experimental data. Furthermore, Fig. 4C shows the multilinear plots of intraparticle
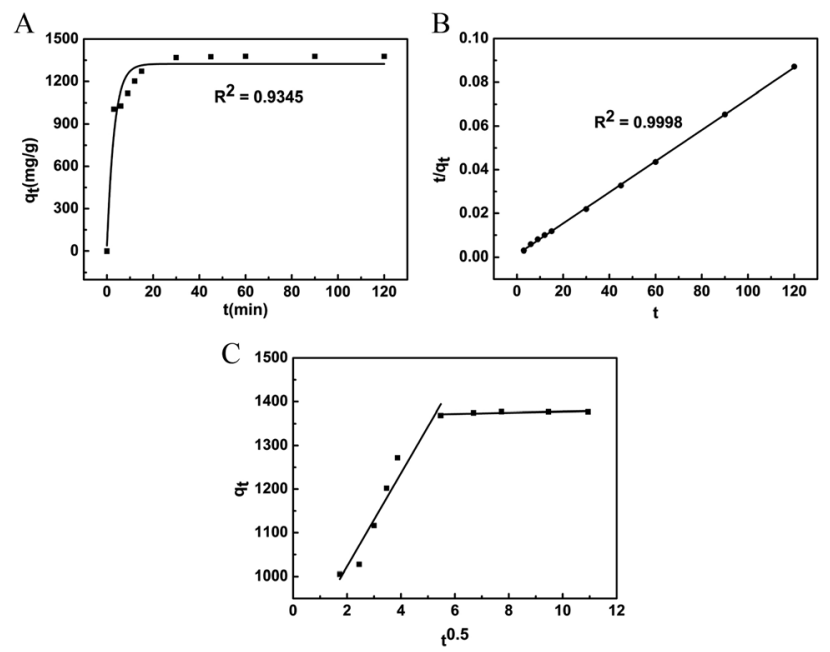

Fig. 4 The fitting of different kinetic models for MB adsorption onto $\mathrm{N}$ O-HPC. (A) Lagergren pseudo-first order, (B) pseudo-second order, (C) intra-particle diffusion. diffusion process of $\mathrm{MB}$ adsorption onto $\mathrm{N}$, O-HPC. It suggests that two steps have taken place during the adsorption process. The first region represents the film diffusion stage which is the diffusion of MB molecules from solution to the external surface of absorbent. And the second region can be ascribed to the intraparticle diffusion stage due to the rough surface and abundant pores of absorbent. ${ }^{78}$ The slope of the film diffusion stage is obviously larger than intraparticle diffusion stage, indicating the intraparticle diffusion stage is a gradual process. Moreover, the plot does not pass through the origin indicating the intraparticle diffusion is not the rate-limiting step and boundary layer phenomenon could also direct the adsorption process.

\subsection{Effect of initial MB concentration and adsorption isotherms}

Fig. $\mathrm{S} 2 \mathrm{~A} \dagger$ is the time dependence of $\mathrm{MB}$ adsorption capacities on N, O-HPC at various initial concentrations. As shown, despite the different initial concentrations, the MB uptake was very rapid and efficient, and adsorption capacity of $\mathrm{MB}$ onto the adsorbent drastically increase during the initial stage and then at a slow speed until reach a state of equilibrium. The results can be ascribed to the fact that most vacant adsorption active sites are available for adsorption during the initial stage and the remaining vacant surface sites are hard to be utilized due to repulsive forces between the $\mathrm{MB}$ molecules on adsorbent and the bulk phase. The equilibrium adsorption capacities of $\mathrm{MB}$ at various initial concentrations demonstrate an increasing trend, since initial $\mathrm{MB}$ concentrations can provide a driving force to overcome the mass transfer resistance of the dye, which indicates that the adsorption process is highly dependent on initial MB concentration. Furthermore, despite various initial concentrations, all the adsorption kinetics can be well fitted by the pseudo-second order model with regression coefficient $R^{2}$ all higher than 0.9996 (Fig. $\mathrm{S} 2 \mathrm{~B} \dagger$ ), which is consistent with the former adsorption kinetic study.

The equilibrium adsorption isotherm was studied in detail, since it could provide information about the surface properties of adsorbent and the adsorption behaviour. In this study, the equilibrium adsorption data is analysed by Langmuir, Freundlich and Temkin models. As shown in Fig. 5, the regression coefficient $R^{2}$ obtained from the Langmuir isotherm model is much higher than those from other models, suggesting that Langmuir isotherm model can describe the experimental data much more suitably, which also indicating the presence of homogenously adsorption active sites on the surface of $\mathrm{N}$, OHPC. Moreover, the maximum monolayer adsorbent capacity value of $2104.29 \mathrm{mg} \mathrm{g}^{-1}$ predicted by the Langmuir model is among the highest in the previously reported adsorbents (Table $\mathrm{S} 1 \dagger$ ), which proves $\mathrm{N}, \mathrm{O}-\mathrm{HPC}$ as an effective adsorbent for MB uptake from aqueous solution. Furthermore, the feasibility of the adsorption process was evaluated using the dimensionless separation factor, the value of RL was found to be in the range of 0.005-0.013, which showed that the adsorption of MB onto N, O-HPC was favourable. 
A
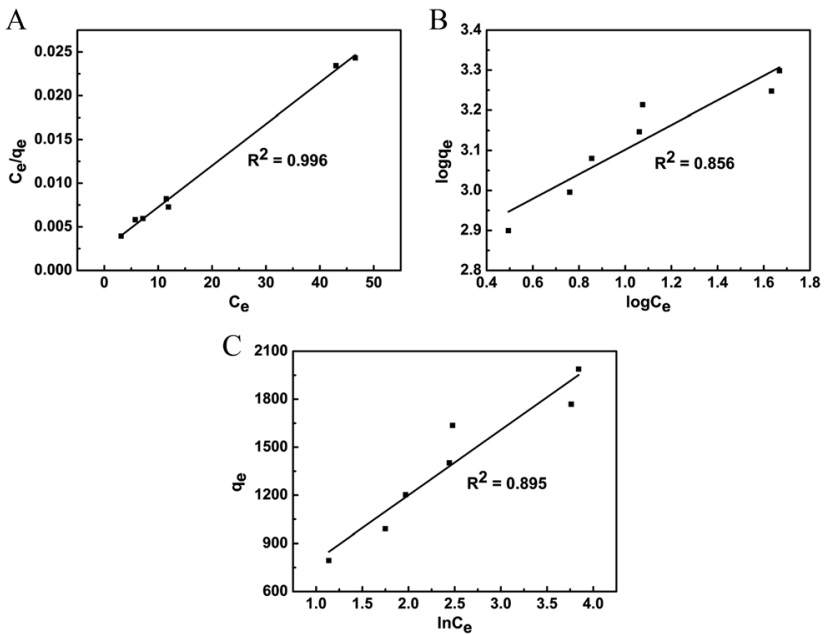

Fig. 5 The fitting of different isotherm models for $M B$ adsorption onto N, O-HPC. (A) Langmuir, (B) Freundlich, (C) Temkin.

\subsection{Effect of temperature and adsorption thermodynamics}

Fig. S3A $\dagger$ shows the time dependence of $\mathrm{MB}$ adsorption capacities on N, O-HPC at different temperatures. During the first $30 \mathrm{~min}$, the adsorption capacity presents a significant increasing trend with the rise in temperature. The equilibrium adsorption capacity increases from 1368 to $1381 \mathrm{mg} \mathrm{g}^{-1}$ as the temperature increases from $293.15 \mathrm{~K}$ to $313.15 \mathrm{~K}$. The results suggest that $\mathrm{MB}$ adsorption on the adsorbent is favoured at higher temperatures within the appropriate temperature range, which may be ascribed to that the mobility of MB molecules increases with the rise in temperature and more dye molecules can interact with the active sites of N, O-HPC.

Thermodynamics parameters including enthalpy change $\left(\Delta H^{0}\right)$, Gibbs free energy change $\left(\Delta G^{0}\right)$ and entropy change $\left(\Delta S^{0}\right)$, enable proper understanding of the inherent energetic changes occurring during adsorption and show whether the reaction can happen spontaneously or not. $\Delta G^{0}$ was determined by the Gibbs isotherm equation $\left(\Delta G^{0}=-R T \ln K_{\mathrm{d}}\right)$, while $\Delta H^{0}$ and $\Delta S^{0}$ were calculated from the slope and intercept of the Van't Hoff plot (Fig. S3B $\dagger$ ). The calculated values were presented in Table S2. $\dagger$ The negative values of $\Delta G^{0}$ confirmed the spontaneous nature and the feasibility of the adsorption process. Besides, the decrease in the negative value of $\Delta G^{0}$ with an increase in temperature suggested the adsorption is more favourable at higher temperature. The positive value of $\Delta H^{0}$ indicated this adsorption process is endothermic, which is consistent with the effect of temperature. The positive value of $\Delta S^{0}$ implied good affinity of MB molecules towards the adsorbent and an increasing randomness at the solid-solution interface during the adsorption process increases. All the thermodynamic parameters mentioned above indicate that $\mathrm{N}$, O-HPC can be used as a high-efficiency adsorbent to remove $\mathrm{MB}$ from aqueous solution.

\subsection{Effect of adsorbent dosage}

Fig. S4A $\dagger$ shows the time dependence of $\mathrm{MB}$ adsorption capacities on N, O-HPC at different adsorbent dosage. As shown, there was a rapid adsorption capacity increase with increasing adsorbent mass dosage from $10 \mathrm{mg}$ to $50 \mathrm{mg}$ and afterwards almost reached a saturation level at higher dosage. This resulted from the creation of additional surface area and adsorption active sites as the adsorbent dosage increases, which enhances MB uptake. Furthermore, all the adsorption kinetics can also be well fitted by the pseudo-second order model with regression coefficient $R^{2}$ all higher than 0.9992 (Fig. $\mathrm{S} 4 \mathrm{~B} \dagger$ ), which is also consistent with the former adsorption kinetic study.

\subsection{Effect of humic acid (HA) and ionic strength}

HA is a common model compound of natural organic matter and widely exists in the aquatic environment. ${ }^{79}$ Effect of HA on MB adsorption was studied (Fig. 6A and B). Negligible impact can be observed when the HA concentrations below $20 \mathrm{mg} \mathrm{L}^{-1}$, while higher HA concentrations gave a negative effect on the adsorption process. The adsorption rate decreased and a much longer time was needed to reach the adsorption equilibrium stage as the HA concentration increased from 20 to $160 \mathrm{mg} \mathrm{L}^{-1}$. Moreover, the remove efficiency decreased from $97.69 \%$ to $91.48 \%$ as the HA concentration increased from 20 to $160 \mathrm{mg} \mathrm{L}^{-1}$. The reduction of adsorption rate and remove efficiency could be resulted from the competitive adsorption between HA and MB molecules toward the adsorbent. ${ }^{80}$ Actually, the HA concentration in realistic water environment was much lower than that of in this study, implying that N, O-HPC exhibited excellent anti-interference ability and had potential to be used in real water matrix on MB removal.

The impact of ionic strength was investigated using $\mathrm{NaCl}$ with different concentrations. Generally, ionic strength has two potential impacts in organic contaminants adsorption by carbonaceous adsorbents. On the one hand, increasing ionic strength can enhance the activity coefficient of hydrophobic
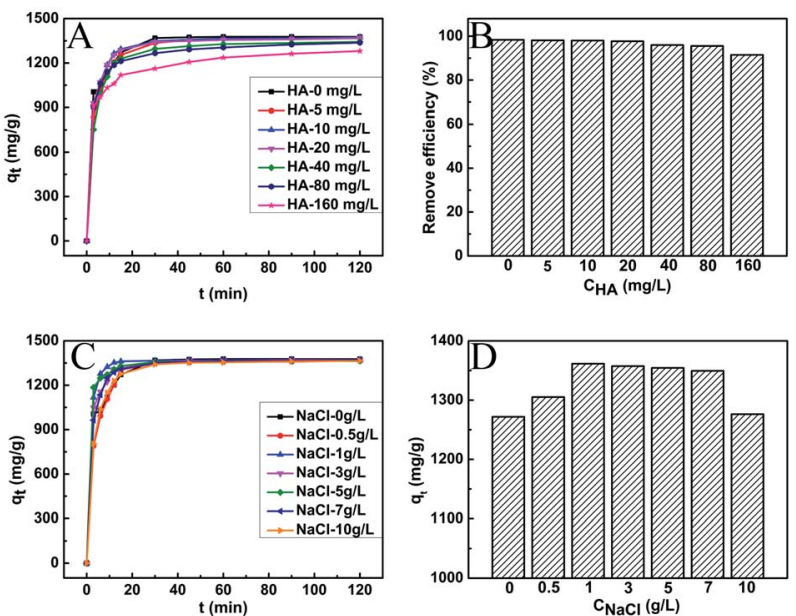

Fig. 6 Effect of HA concentration and ionic strength on ( $A$ and $C$ ) the time-dependent adsorption capacity, (B) the equilibrium remove efficiency and (D) the remove efficiency at 15 min of $M B$ onto N, O$\mathrm{HPC}\left([\mathrm{MB}]=700 \mathrm{mg} \mathrm{L}^{-1}\right.$, absorbent dosage $\left.=0.05 \mathrm{~g}, 293 \mathrm{~K}\right)$. 
organic compounds, leading to a decrease in their solubility (i.e. salting out effect), which is favourable for organic contaminants adsorption. ${ }^{81}$ Besides, the increase of ionic strength give rise to the compression of the diffuse double layer on the adsorbent, which facilitated the electrostatic attraction and contributed to the adsorption consequently. ${ }^{82}$ On the other hand, the increase in salt ions can exert a "squeeze-out" effect on carbonaceous adsorbents, which is unfavourable for organic contaminants adsorption. ${ }^{83,84}$ As shown in Fig. 6C, the equilibrium adsorption capacities basically kept unchanged even the salt concentrations were up to $10 \mathrm{~g} \mathrm{~L}^{-1}$. However, the variation of $\mathrm{NaCl}$ concentration exhibited a significant effect on the adsorption rate and high salt concentration was contributive to dye removal, especially in the first $15 \mathrm{~min}$ (Fig. 6D). This facilitation for MB adsorption could be resulted from both the salting-out effect and the enhanced electrostatic attraction.

\subsection{Effect of initial solution $\mathrm{pH}$}

The $\mathrm{pH}$ is one of the most significant environmental factors influencing the adsorption process. Solution $\mathrm{pH}$ would influence both aqueous chemistry and surface binding sites of the adsorbents. The pHpzc is very crucial to judge the surface charge of adsorbents. The surface of the adsorbent is negatively charged at $\mathrm{pH}>\mathrm{pHpzc}$, or it is positively charged at $\mathrm{pH}<\mathrm{pHpzc}$. The pHpzc of N, O-HPC was determined by the classical immersion technique and found to be 7.3 (Fig. 7A). To study the influence of initial solution $\mathrm{pH}$ on the adsorption capacities of N, O-HPC for MB, experiments were carried out using various pHs varying from 1 to 9 . From Fig. $7 \mathrm{~B}$, it is observed that the adsorption was highly dependent on solution $\mathrm{pH}$, and the dye adsorption capacities increased with the rise of solution $\mathrm{pH}$. At low $\mathrm{pH}(\leq 7)$, the dye adsorption capacities decreased in the order: $1>3>7$, which was ascribed to the surface of the adsorbent was positively charged, which generated repulsive forces between $\mathrm{MB}^{+}$cations and positively charged adsorption sites triggering decline in dye removal. Moreover, the competition between excess amount of $\mathrm{H}^{+}$ions and $\mathrm{MB}^{+}$cations for negatively charged adsorption sites also reduced the adsorption capacities. At $\mathrm{pH}=9$, the negatively charged adsorption sites on adsorbents were enriched causing high electrostatic attraction between $\mathrm{MB}^{+}$and adsorbent, leading to enhanced adsorption capacities. Similar results can be found in previous literatures.
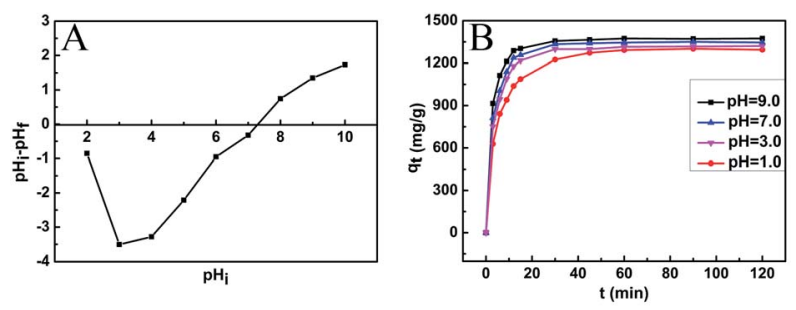

Fig. 7 (A) pHpzc of $\mathrm{N}, \mathrm{O}-\mathrm{HPC}$, (B) Effect of initial solution $\mathrm{pH}$ on the time-dependent adsorption capacity of MB onto $\mathrm{N}, \mathrm{O}-\mathrm{HPC}([\mathrm{MB}]=$ $700 \mathrm{mg} \mathrm{L}^{-1}$, absorbent dosage $=0.05 \mathrm{~g}, 293 \mathrm{~K}$ ).

\subsection{Adsorption mechanism}

Fourier transform infrared (FTIR) spectra of N, O-HPC, MB adsorbed N, O-HPC and pure MB were performed to gain more insights into the adsorption mechanism. FTIR spectrum of N, O-HPC (Fig. 8A) confirmed the presence of $-\mathrm{OH}\left(3435 \mathrm{~cm}^{-1}\right)$, $-\mathrm{C}=\mathrm{C}-\left(1635 \mathrm{~cm}^{-1}\right), \mathrm{C}=\mathrm{O} / \mathrm{C}=\mathrm{N}\left(1589 \mathrm{~cm}^{-1}\right)$ and $\mathrm{C}-\mathrm{N} / \mathrm{C}-\mathrm{O}$ $\left(1120 \mathrm{~cm}^{-1}\right)$, which led to the hydrophilic nature of N, O-HPC and acted as anchoring sites for MB molecules. In the case of MB (Fig. 8C), the peak at $1597 \mathrm{~cm}^{-1}$ was assigned to the stretching vibrations of $\mathrm{C}=\mathrm{N}$ ( and $\mathrm{C}=\mathrm{C}$ ) bond in the heterocycle of $\mathrm{MB}$, while the peaks at 1350 and $1329 \mathrm{~cm}^{-1}$ were attributed to the stretching vibration of $\mathrm{C}-\mathrm{N}$ bond connected with benzene ring and $\mathrm{N}-\mathrm{CH}_{3}$ bond. The peak at about $1487 \mathrm{~cm}^{-1}$ was ascribed to the $\mathrm{CH}_{2}$ deformation vibration, while the peak around $1388 \mathrm{~cm}^{-1}$ was owing to the $\mathrm{CH}_{3}$ deformation vibration. Furthermore, the peaks at about 1246 and $1217 \mathrm{~cm}^{-1}$ were due to $\mathrm{Ar}-\mathrm{N}$ deformation vibration, the peaks at 1168 and $1136 \mathrm{~cm}^{-1}$ to the stretching vibrations of $\mathrm{C}=\mathrm{S}$ and $\mathrm{C}-\mathrm{S}$, and the band at about $884 \mathrm{~cm}^{-1}$ to the wagging vibration of $\mathrm{C}-\mathrm{H}$ in aromatic ring of MB. After adsorption of MB (Fig. 8B), the peak at $3435 \mathrm{~cm}^{-1}$ of N, O-HPC remained unchanged, which indicated that hydrogen bonding may not be an important process for MB adsorption onto N, O-HPC. However, the new absorption peak at 1383 and $880 \mathrm{~cm}^{-1}$ could be clearly observed, indicating that MB has been anchored on the surface of N, O-HPC during the adsorption process. Moreover, after absorbed by N, O-HPC, obvious shifts were observed to $1593 \mathrm{~cm}^{-1}$ for $\mathrm{C}=\mathrm{N}$ (and $\mathrm{C}=\mathrm{C}$ ) bond in the heterocycle of $\mathrm{MB}$, to $1455 \mathrm{~cm}^{-1}$ for $\mathrm{CH}_{2}$ deformation vibration in benzene ring and to $880 \mathrm{~cm}^{-1}$ for the wagging vibration of $\mathrm{C}-\mathrm{H}$ in aromatic ring of $\mathrm{MB}$, which corresponded to the attachment of MB on the surface of N, O-HPC by $\pi-\pi$ stacking interaction between the aromatic backbone of MB and hexagonal skeleton of N, O-HPC since MB was an ideally planar molecule. ${ }^{85,86}$ Besides, the electronegativity of $\mathrm{N}$ is

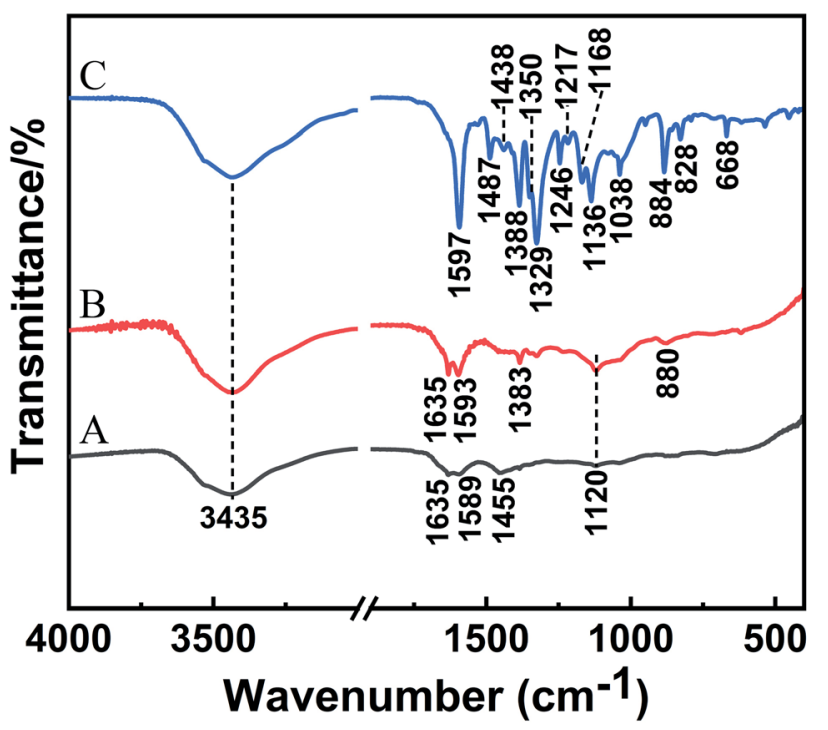

Fig. 8 FTIR spectra of N, O-HPC before (A) and after (B) adsorption of $M B$ and pure $M B(C)$. 

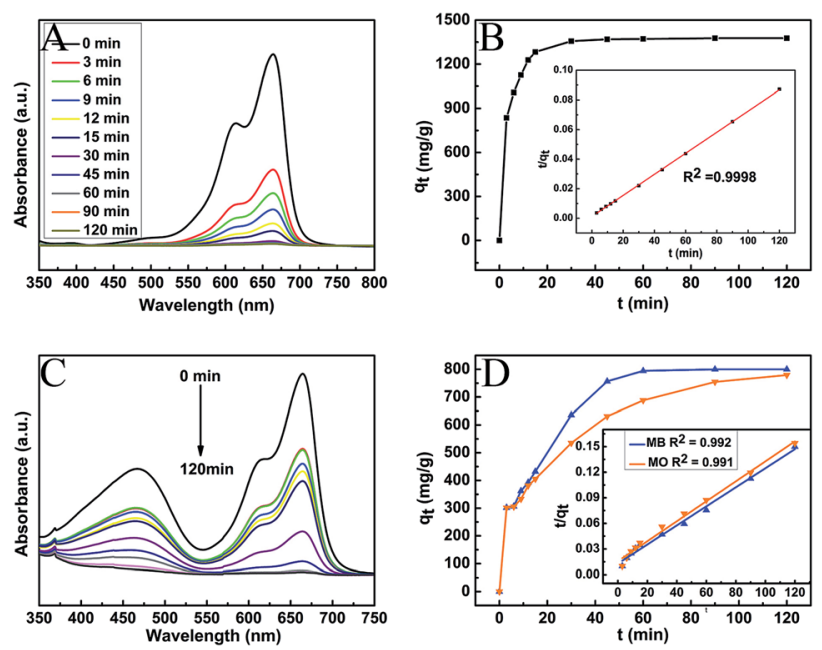

Fig. 9 (A) Time-dependent UV-vis absorption spectra of MB solutions, (B) effect of contact time on MB adsorption capacities. Inset: corresponding pseudo-second order model fit $\left([\mathrm{MB}]=700 \mathrm{mg} \mathrm{L}^{-1}\right.$ absorbent dosage $=0.05 \mathrm{~g}, 293 \mathrm{~K}$, solvent: tap water), (C) timedependent UV-vis absorption spectra of $\mathrm{MB}$ and $\mathrm{MO}$ solutions, (D) effect of contact time on $\mathrm{MB}$ and $\mathrm{MO}$ adsorption capacities and their corresponding pseudo-second order kinetic model fittings ([MB] = $[\mathrm{MO}]=400 \mathrm{mg} \mathrm{L}^{-1}$, absorbent dosage $=0.05 \mathrm{~g}, 293 \mathrm{~K}$ )

larger than that of $\mathrm{C}(\chi \mathrm{N}(3.04)>\chi \mathrm{C}(2.55))$, the electronegative graphitic $\mathrm{N}$ atoms could positively polarize their neighbouring carbon atoms, thus the corresponding negatively charged graphitic $\mathrm{N}$ atoms were able to act as adsorption sites to absorb cationic MB via electrostatic attraction. Based on the above, the exceptionally high uptake of MB onto N, O-HPC resulted from not only high specific surface area and abundant mesopores/ micropores of N, O-HPC, but also multiple adsorption mechanisms including pore filling, $\pi-\pi$ stacking interaction and electrostatic interaction between MB and N, O-HPC.

\subsection{Adsorption of MB in tap water and synergistic adsorption of MB and MO}

As indicated in Fig. 9A and B, despite in tap water solution, the maximum adsorption peak at $664 \mathrm{~nm}$ of $\mathrm{MB}$ gradually decreased with the increase of time and the adsorption process reached equilibrium state at about $30 \mathrm{~min}$. Furthermore, the as prepared adsorbent showed synergistic adsorption performance for MB and methyl orange (MO). As shown in Fig. 9C, the maximum adsorption peak at $464 \mathrm{~nm}$ of $\mathrm{MO}$ and at $664 \mathrm{~nm}$ of MB both gradually decreased as the rise of time. Furthermore, all the adsorption kinetics can also be well fitted by the pseudosecond order model with regression coefficient $R^{2}$ all higher than 0.99 (Fig. 9D). These results indicated N, O-HPC could be considered as efficient, novel and advantageous adsorbent for the removal of both cationic and anionic dyes.

\section{Conclusions}

In summary, $\mathrm{N}$, O-codoped hierarchical porous carbon $(\mathrm{N}, \mathrm{O}-$ HPC) derived from silkworm cocoon with an ultrahigh specific surface area of $2270.19 \mathrm{~m}^{2} \mathrm{~g}^{-1}$ was successfully synthesized via a facile pre-carbonization and chemical activation method, and characterized thoroughly. The as-prepared N, O-HPC proved to be a high-efficiency adsorbent. Batch adsorption experimental results demonstrated that the MB adsorption was highly dependent on contact time, initial MB concentration, temperature and initial solution $\mathrm{pH}$, while no remarkable effects of humic acid and ionic strength are observed. Kinetics experiments clearly indicated the adsorption process followed pseudo-second-order kinetic model. Besides, Weber's intraparticle diffusion model indicated two steps have taken place during the adsorption process and the intraparticle diffusion was not the rate-limiting step. The adsorption isotherm study showed that Langmuir isotherm model can describe the experimental data much more suitably with a maximum monolayer adsorption capacity value of $2104.29 \mathrm{mg} \mathrm{g}^{-1}$, which is among the highest in the previously reported adsorbents and ascribed to multiple adsorption mechanisms including pore filling, $\pi-\pi$ stacking interaction and electrostatic interaction between MB and N, O-HPC. By thermodynamic analysis, it was shown that MB adsorption onto N, O-HPC was spontaneous and endothermic. Furthermore, the as prepared adsorbent showed highly efficient adsorption for $\mathrm{MB}$ in tap water and synergistic adsorption performance toward MB and MO. Therefore, N, OHPC derived from silkworm cocoon can be developed as an efficient, novel and advantageous material for wastewater remediation.

\section{Conflicts of interest}

We have no competing interests.

\section{Acknowledgements}

The authors would like to thank Huili Liu from Huanghe Science \& Technology College and Lijie Hou from Shiyanjia Lab (www.shiyanjia.com) for their assistance in material characterizations. This work was supported by Special Funding Project for Basic Scientific Research Operation Expenses (K2018QN021) and Program for Interdisciplinary Direction Team in Zhongyuan University of Technology, and sponsored by Program for Science and Technology Innovation Talents in Universities of Henan Province (No. 19HASTIT024).

\section{Notes and references}

1 A. M. M. Vargas, A. L. Cazetta, M. H. Kunita, T. L. Silva and V. C. Almeida, Chem. Eng. J., 2011, 168, 722-730.

2 O. J. Hao, H. Kim and P.-C. Chiang, Crit. Rev. Environ. Sci. Technol., 2000, 30, 449-505.

3 D. Zhao, G. Sheng, C. Chen and X. Wang, Appl. Catal., B, 2012, 111-112, 303-308.

4 Y. L. Min, K. Zhang, W. Zhao, F. C. Zheng, Y. C. Chen and Y. G. Zhang, Chem. Eng. J., 2011, 193-194, 203-210.

5 I. K. Konstantinou and T. A. Albanis, Appl. Catal., B, 2004, 49, $1-14$. 
6 U. G. Akpan and B. H. Hameed, J. Hazard. Mater., 2009, 170, 520-529.

7 M. Panizza, A. Barbucci, R. Ricotti and G. Cerisola, Sep. Purif. Technol., 2007, 54, 382-387.

8 A. I. del Río, C. García, J. Molina, J. Fernández, J. Bonastre and F. Cases, Chemosphere, 2017, 183, 242-251.

9 I. Fatimah, S. Wang and D. Wulandari, Appl. Clay Sci., 2011, 53, 553-560.

10 V. K. Gupta, R. Jain, A. Mittal, M. Mathur and S. Sikarwar, J. Colloid Interface Sci., 2007, 309, 464-469.

11 C. Minero, M. Lucchiari, D. Vione and V. Maurino, Environ. Sci. Technol., 2005, 39, 8936-8942.

12 S. Merouani, O. Hamdaoui, F. Saoudi and M. Chiha, Chem. Eng. J., 2010, 158, 550-557.

13 M. Abbasi and N. R. Asl, J. Hazard. Mater., 2008, 153, 942947.

14 Y. Areerob, J. Y. Cho, W. K. Jang and W.-C. Oh, Ultrason. Sonochem., 2018, 41, 267-278.

15 L. Nirumand, S. Farhadi, A. Zabardasti and A. Khataee, Ultrason. Sonochem., 2018, 42, 647-658.

16 G. Zhou, Z. Chen, F. Fang, Y. He, H. Sun and H. Shi, J. Environ. Sci., 2015, 35, 20-26.

17 M. E. Farshchi, A. Hassan and A. Khataee, J. Clean. Prod., 2018, 182, 644-653.

18 X. Shi, A. Tian, J. You, H. Yang, Y. Wang and X. Xue, J. Hazard. Mater., 2018, 353, 182-189.

19 Y. Liu, W. Jin, Y. Zhao, G. Zhang and W. Zhang, Appl. Catal., $B, 2017,206,642-652$.

20 L. Zhou, J. Lei, L. Wang, Y. Liu and J. Zhang, Appl. Catal., B, 2018, 237, 1160-1167.

21 H. Lan, A. Wang, R. Liu, H. Liu and J. Qu, J. Hazard. Mater., 2015, 285, 167-172.

22 G. Antoine, A. M. Tuqan, N. Kibbi and S. Geryes, Chem. Eng. J., 2012, 213, 259-271.

23 C.-H. Weng and H. Tao, Arabian J. Chem, 2018, 11, 12921300.

24 S. Wacławek, H. V. Lutze, K. Grübel and V. V. T. Padil, Chem. Eng. J., 2017, 330, 44-62.

25 S. Bo, J. Luo, Q. An, X. Zhao, Z. Xiao, S. Zhai and Z. Li, J. Clean. Prod., 2019, 236, 117630.

26 C. Flox, S. Ammar, C. Arias, E. Brillas, A. ViridianaVargasZavala and R. Abdelhedi, Appl. Catal., B, 2006, 67, 93-104.

27 G. Xia, Y. Lu, X. Gao, C. Gao and H. Xu, Clean. - Soil, Air, Water, 2015, 43, 229-236.

28 S. A. Ali, I. Y. Yaagoob, M. A. J. Mazumder and H. A. AlMuallem, J. Hazard. Mater., 2019, 369, 642-654.

29 A. M. Ghaedi and A. Vafaei, Adv. Colloid Interface Sci., 2017, 245, 20-39.

30 G. Ersan, O. G. Apul, F. Perreault and T. Karanfil, Water Res., 2017, 126, 385-398.

31 A. Ali Siyal, M. Rashid Shamsuddin, M. I. Khan, N. E. Rabat, M. Zulfiqar, Z. Man, J. Siame and K. A. Azizli, J. Environ. Manage., 2018, 224, 327-339.

32 G. Z. Kyzas, E. A. Deliyanni, D. N. Bikiaris and A. C. Mitropoulos, Chem. Eng. Res. Des., 2018, 129, 75-88.
33 D. Jiang, M. Chen, H. Wang, G. Zeng, D. Huang, M. Cheng, Y. Liu, W. Xue and ZiW. Wang, Coord. Chem. Rev., 2019, 380, 471-483.

34 J. Lv, S. Zhai, Y. Fan, Z. Lei and Q. An, J. Taiwan Inst. Chem. Eng., 2016, 62, 209-218.

35 Y. Chen, S.-R. Zhai, N. Liu, Y. Song, A. Qing-Da and X.-W. Song, Bioresour. Technol., 2013, 144, 401-409.

36 I. Ali and V. K. Gupta, Nat. Protoc., 2007, 1, 2661-2667.

37 M. Rafatullah, O. Sulaiman, R. Hashim and A. Ahmad, J. Hazard. Mater., 2010, 177, 70-80.

38 I. Ali, M. Asim and T. A. Khan, J. Environ. Manage., 2012, 113, 170-183.

39 B. Amit, M. Sillanpää and A. Witek-Krowiak, Chem. Eng. J., 2015, 270, 244-271.

40 B. $\mathrm{Mu}$ and A. Wang, J. Environ. Chem. Eng., 2016, 4, 12741294.

41 R. Yang, D. Li, A. Li and H. Yang, Appl. Clay Sci., 2018, 151, 20-28.

42 T. Ngulube, J. R. Gumbo, V. Masindi and A. Maity, J. Environ. Manage., 2017, 191, 35-57.

43 A. Kausar, M. Iqbal, A. Javed, K. Aftab, Z.-i-H. Nazli, H. N. Bhatti and S. Nouren, J. Mol. Liq., 2018, 256, 395-407.

44 I. Anastopoulos and G. Z. Kyzas, J. Mol. Liq., 2014, 200, 381389.

45 A. H. Shalla, M. A. Bhat and Z. Yaseen, J. Environ. Chem. Eng., 2018, 6, 5938-5949.

46 P. M. Pakdel and S. J. Peighambardoust, Carbohydr. Polym., 2018, 201, 264-279.

47 P. M. Pakdel and S. J. Peighambardoust, J. Environ. Manage., 2018, 217, 123-143.

48 V. K. Gupta, R. Kumar, A. Nayak, T. A. Saleh and M. A. Barakat, Adv. Colloid Interface Sci., 2013, 193-194, 24-34.

49 S. Senthilkumaar, P. R. Varadarajan, K. Porkodi and C. V. Subbhuraam, J. Colloid Interface Sci., 2005, 284, 78-82.

50 D. E. Chung, H. H. Kim, M. K. Kim, K. H. Lee, Y. H. Park and I. C. Um, Int. J. Biol. Macromol., 2015, 79, 943-951.

51 F. Chen, D. Porter and V. Fritz, Acta Biomater., 2012, 8, 26202627.

52 N. Fiol and I. Villaescusa, Environ. Chem. Lett., 2009, 7, 7984.

53 S. Tangsir, L. D. Hafshejani, L. Anna, M. Maljanen, A. Hooshmand, A. Ali Naseri, H. Moazed, J. Jokiniemi and B. Amit, Chem. Eng. J., 2016, 288, 198-206.

54 S. Lagergren, K. Sven. Vetenskapsakad. Handl., 1898, 24, 1-39. 55 Y. S. Ho and G. McKay, Process Biochem., 1999, 34, 451-465. 56 W. J. Weber Jr and J. C. Morris, J. Sanit. Eng. Div. Proc. Am.

Soc. Civ. Eng., 1963, 89, 31-60.

57 I. Langmuir, J. Am. Chem. Soc., 1916, 38, 2221-2295.

58 I. Langmuir, J. Am. Chem. Soc., 1918, 40, 1361-1403.

59 H. M. F. Freundlich, Z. Phys. Chem., 1906, 57, 385-470.

60 M. I. Temkin, Zh. Fiz. Khim., 1941, 15, 296-332.

61 O. Pezoti, A. L. Cazetta, K. C. Bedin, L. S. Souza, A. C. Martins, T. L. Silva, O. O. Santos Júnior, J. V. Visentainer and V. C. Almeida, Chem. Eng. J., 2016, 288, 778-788. 
62 D. He, Z. Wang, P. Lia, Z. Liu, H. Wu, L. Liu, K. Han, L. Liu, Q. Wan, F. K. Butt and X. Qu, Appl. Surf. Sci., 2019, 465, 303312.

63 F. Su, C. K. Poh, J. S. Chen, G. Xu, D. Wang, L. Qin, J. Lin and X. W. Lou, Energy Environ. Sci., 2011, 4, 717-724.

64 J. P. Paraknowitsch, J. Zhang, D. Su, A. Thomas and M. Antonietti, Adv. Mater., 2010, 22, 87-92.

65 G. Zhao, C. Chen, D. Yu, L. Sun, C. Yang, H. Zhang, Y. Sun, F. Besenbacher and M. Yu, Nano Energy, 2018, 47, 547-555.

66 A. C. Ferrari and J. Robertson, Phys. Rev. B: Condens. Matter Mater. Phys., 2000, 61, 14095-14107.

67 L. M. Malard, M. A. Pimenta, G. Dresselhaus and M. S. Dresselhaus, Phys. Rep., 2009, 473, 51-87.

68 H. Ait Ahsaine, M. Zbair, Z. Anfar, Y. Naciri, R. El haouti, N. El Alem and M. Ezahri, Mater. Today Chem., 2018, 8, 121-132.

69 A. C. Ferrari and D. M. Basko, Nat. Nanotechnol., 2013, 8, 235-246.

70 J. Wang and S. Kaskel, J. Mater. Chem., 2012, 22, 2371023725.

71 P. Deng, S. Lei, W. Wang, W. Zhou, X. Ou, L. Chen, Y. Xiao and B. Cheng, J. Mater. Sci., 2018, 53, 14536-14547.

72 Y. Li, Y. Zhao, H. Cheng, Y. Hu, G. Shi, L. Dai and L. Qu, J. Am. Chem. Soc., 2012, 134, 15-18.

73 Q. Long, W. Chen, H. Xu, X. Xiong, Y. Jiang, F. Zou, X. Hu, Y. Xin, Z. Zhang and Y. Huang, Energy Environ. Sci., 2013, 6, 2497-2504.
74 J. Casanovas, J. M. Ricart, J. Rubio, F. Illas and J. M. JiménezMateos, J. Am. Chem. Soc., 1996, 118, 8071-8076.

75 T. Lin, I.-W. Chen, F. Liu, C. Yang, B. Hui, F. Xu and F. Huang, Science, 2015, 350, 1508-1513.

76 L. Jing, W. Liu, D. Xiao and X. Wang, Appl. Surf. Sci., 2017, 416, 918-924.

77 L. T. Popoola, T. A. Aderibigbe, A. S. Yusuff and M. Mofehintoluwa Munir, Environ. Qual. Manag., 2018, 28, 63-78.

78 J. Fu, Z. Chen, M. Wang, S. Liu, J. Zhang, J. Zhang, R. Han and Q. Xu, Chem. Eng. J., 2015, 259, 53-61.

79 G. Z. Kyzas, D. N. Bikiaris and D. A. Lambropoulou, J. Mol. Liq., 2017, 230, 1-5.

80 Y.-Y. Cui, Hu-B. Ren, C.-X. Yang and X.-P. Yan, Chem. Eng. J., 2019, 368, 589-597.

81 L.-h. Jiang, Y.-g. Liu, G.-m. Zeng, F.-y. Xiao, X.-j. Hu, X. Hu, H. Wang, T.-t. Li, L. Zhou and X.-f. Tan, Chem. Eng. J., 2016, 284, 93-102.

82 S. Zhang, S. Ting Shao, S. K. Bekaroglu and T. Karanfil, Water Res., 2010, 44, 2067-2074.

83 X. Zhu, D. C. W. Tsang, F. Chen, S. Li and X. Yang, Environ. Technol., 2015, 36, 3094-3102.

84 S. Zhang, T. Shao, H. Selcen Kose and T. Karanfil, Environ. Sci. Technol., 2010, 44, 6377-6383.

85 J. Gong, J. Liu, Z. Jiang, X. Wen, E. Mijowska, T. Tang and X. Chen, J. Colloid Interface Sci., 2015, 445, 195-204.

86 L. Ai, C. Zhang, F. Liao, Y. Wang, M. Li, L. Meng and J. Jiang, J. Hazard. Mater., 2011, 198, 282-290. 